\title{
A New Method for Quantifying the Response of Filters at Corners
}

\author{
Mark A. Schulze and John A. Pearce \\ Department of Electrical and Computer Engineering and Biomedical Engineering Program \\ The University of Texas at Austin \\ Austin, Texas 78712
}

\begin{abstract}
$\underline{\text { ABSTRACT }}$
Although the edge response of nonlinear filters has been the topic of much research, the behavior of many filters at two-dimensional structures such as sharp corners is not as well understood. We introduce a new technique that defines the response of a two-dimensional filter at corners of any angle. The response measure may be expressed either as fractional preservation or as an attenuation in decibels. Plotting the response measure in polar form illustrates the corner response of a filter both intuitively and quantitatively. The corner response may be computed for a particular size and shape of filter window on a discrete lattice, or may be determined in continuous space for a filter with a specific window shape. The continuous space response measure gives the response of the filter to corners in general, and usually corresponds closely with that determined for the filter on a discrete lattice. The corner responses of several widely used filters (median, averaging, and morphological) are compared.
\end{abstract}

\section{INTRODUCTION}

The response of one- and two-dimensional filters to edges has been a topic of much research [1-3]. The edge response of nonlinear filters such as the median filter is one of the primary reasons such filters have become important in signal and image processing applications. However, the edge response of a filter tells us little about its response to two-dimensional structures such as corners. For example, the median filter is noted for its exact preservation of edges and elimination of impulsive noise, but it noticeably rounds off sharp corners. We have developed a technique to measure the degree of preservation of corners of all angles by a filter with a given window shape. This technique may be applied to discrete filter windows acting on digital images, or may be used to derive theoretical results in continuous space that yield more general information about filters and window shapes. These corner responses may then be used to help select an appropriate filter for a particular image processing application. The corner responses may also be derived for corners oriented at any angle relative to the Cartesian image axes.

\section{TECHNIQUE}

\subsection{Fractional Corner Preservation}

A corner of angle $\theta$ is defined in 2-D space at the origin of the $x-y$ plane, as shown in Fig. 1, rotated with respect to the $\mathrm{x}$-axis by an angle $\alpha$. The original image, $\mathrm{f}(x, y)$ of the corner consists of only two values; in Fig. 1, white is assumed to be 0 and black to be 1 . We will assume $\theta$ takes on values in the range $-\pi<\theta \leq \pi$. The regions I, II, III, and IV in Fig. 1 are each the size of the smallest square that the overall filter window will fit in. The total area of the corner defined by $\theta$ and $\alpha$ is defined by the area within the angle $\theta$ that is also within regions I, II, III, or IV. Thus in Fig. 1, the total corner area, $A$, is the black area inside region I. Since $\mathrm{f}(x, y)=1$ everywhere in $A$,

$$
\int_{A} \mathrm{f}(x, y)=A .
$$

\footnotetext{
Copyright 1994 Society of Photo-Optical Instrumentation Engineers.

This paper was published in E. R. Dougherty, J. Astola, and H. Longbotham, eds., Nonlinear Image Processing V, Proc. SPIE, v. 2180, pp. 78-89, and is made available as an electronic reprint with permission of SPIE. One print or electronic copy may be made for personal use only. Systematic or multiple reproduction, distribution to multiple locations via electronic or other means, duplication of any material in this paper for a fee or for commercial purposes, or modification of the content of the paper are prohibited.
} 
Filtering usually changes parts of the corner from black (1) to gray or white (values less than 1). This is illustrated in Fig. 2. If $\mathrm{g}(x, y)$ denotes the filtered image, then we define the preserved corner area $P$ as:

$$
P=\int_{A} \mathrm{~g}(x, y) .
$$

For the median filter and other filters that do not return intermediate values for a bi-valued input, $P$ is the area of $A$ that the filter preserves as black (pixel value 1). For filters that do yield intermediate values, such as the averaging and other linear filters, $P$ corresponds to the relative amplitude preservation of the corner only in the area defined by the original corner.

The fractional preservation of a corner of angle $\theta$ oriented at an angle $\alpha$ with respect to the $\mathrm{x}$-axis, denoted $\mathrm{r}(\theta, \alpha)$, is defined as:

$$
\mathrm{r}(\theta, \alpha)=\frac{P}{A}
$$

The response of a filter to corners of all angles at a particular orientation $\alpha$ is therefore only a function of the angle $\theta, r(\theta)$. Note that $r(\theta)$ ranges in value from 1 (perfect corner preservation) to 0 (complete removal of the corner in the region of interest).

\subsection{Attenuation}

The fractional preservation can also be expressed as an attenuation in decibels, similar to the usual description of the response of an electrical filter. The corner attenuation in $\mathrm{dB}, \mathrm{s}(\theta, \alpha)$, is defined as:

$$
\mathrm{s}(\theta, \alpha)=20 \log [\mathrm{r}(\theta, \alpha)]=20 \log \left(\frac{P}{A}\right) .
$$

From this definition, $\mathrm{s}(\theta, \alpha)=0 \mathrm{~dB}$ corresponds to perfect corner preservation, and $\mathrm{s}(\theta, \alpha)=-3 \mathrm{~dB}$ corresponds to an average fractional corner preservation of $\frac{1}{\sqrt{2}}$ (that is, about $71 \%$ of the area is preserved intact, or the entire area is preserved at about $71 \%$ amplitude).

\subsection{Continuous or Discrete Space}

The responses $\mathrm{r}(\theta, \alpha)$ and $\mathrm{s}(\theta, \alpha)$ may be determined either for discrete filters acting on a discrete lattice (that is, $x$ and $y$ are integers) or for continuous filters in continuous space ( $x$ and $y$ are real). In the discrete case, each point $(x, y)$ in the lattice corresponds to an angle $\phi$ that is the arctangent of $(y / x)$, and the total corner area $A$ of an angle $\theta$ at orientation $\alpha$ (as in Fig. 1) is assumed to be all points $(x, y)$ in the regions I, II, III and IV where $\alpha=\phi=(\alpha+\theta)$.

For continuous space, equations (1)-(3) apply directly, but the filters must be defined in continuous space. Many filters, such as the averaging and morphological filters, are easily defined in continuous space; others, such as the median filter, have a slightly more subtle generalization [4]. We have found that the response of a discrete filter is typically very similar to that of its corresponding continuous filter, especially for large window sizes. Very large discrete windows give a numerical approximation of the continuous space integrals in equations (1) and (2).

\section{EXAMPLES}

We can use the corner response functions $r(\theta, \alpha)$ and $s(\theta, \alpha)$ to illustrate the behavior of various linear and nonlinear filters at corners. For many filters, such as the averaging and median filter, closed form solutions for $\mathrm{r}(\theta, \alpha)$ and $\mathrm{s}(\theta, \alpha)$ are difficult to derive, even for $\alpha=0$. However, values of $\mathrm{r}$ and $\mathrm{s}$ at particular values of $\theta$ and $\alpha$ 
often are readily found, such as $\theta=45^{\circ}, 90^{\circ}, 135^{\circ}$, and $180^{\circ}$. These values can be compared with the results of discrete simulations with large window sizes to verify the accuracy of the numerical approximation.

\subsection{Response of Morphological Filters}

It is easier to solve $\mathrm{r}(\theta, \alpha)$ and $\mathrm{s}(\theta, \alpha)$ for the filtering operations of mathematical morphology. This is because the morphological filters are geometrical operations, and therefore their response can usually be determined by the interaction of the filter window shape with the corner. Morphological erosion is simply a sliding minimum operator acting on an image with a filter window, called a "structuring element." Morphological dilation is a

sliding maximum operator. For binary images, the erosion of an image has values of one only in areas of the original image where the structuring element fits entirely within a region of ones. The dilation of a binary image is similar, except that it only has zeroes in areas of the original for which the structuring element fits completely within a zero region. The compound morphological operators open and close are, respectively, erosion followed by dilation and dilation followed by erosion. The doubly compound operations are open-close (OC), which is opening followed by closing, and close-open (CO), which is closing followed by opening.

Maragos and Schafer [5] have shown that to compare the compound morphological operations to other filters, particularly the median filter, the overall filter window is found by compounding the morphological structuring element with its $180^{\circ}$ rotation. This makes sense because opening and closing draw their potential output values from this area, since these operations make two passes with the structuring element. Between passes, there is $180^{\circ}$ rotation of the structuring element, which is usually ignored since most structuring elements are symmetric about the origin. For example, a $3 \times 3$ structuring element compounded with itself results in a $5 \times 5$ square overall filter window. In continuous space, a square structuring element with sides of length $l$ compounds to a square with sides of length 2 . The regions of interest (I, II, III, and IV) as in Fig. 1 are the smallest squares in which the overall window (the compounded structuring element) will fit.

Consider the case of a square structuring element of arbitrary size. In continuous space, the fractional corner preservation for the morphological closing is unity for all $\theta$ and $\alpha$; that is, $r(\theta, \alpha)=1$. The corner attenuation is therefore $s(\theta, \alpha)=0$ for all angles $\theta$ and rotations $\alpha$. We derive this result by noting that even for the sharpest corners, the square dilation expands them so that the subsequent erosion returns them to their original shape.

However, morphological opening does tend to clip off sharp corners because its first operation is erosion. Opening preserves high values only at pixels where an entire structuring element including that pixel is filled with high values. By simple geometry, closed form solutions for $\mathrm{r}(\theta, \alpha)$ and $\mathrm{s}(\theta, \alpha)$ may be obtained for opening. For $\alpha=0$ and $\theta<\tan ^{-1}(0.5)$, roughly $27^{\circ}$, the square structuring element does not fit even partially within both the corner and region I, so $r(\theta)=0$. (Recall that for opening, the structuring element is compounded to form the overall window. Therefore, the structuring element is half as wide and half as tall as region I.) The complete expression for $r(\theta, \alpha)$ when $\alpha=0$ for opening with a square structuring element is:

$$
\begin{array}{rlrl}
\mathrm{r}_{\text {open }}(\theta, 0)= & 0, & 0 \leq|\theta|<\tan ^{-1}\left(\frac{1}{2}\right) \\
& 1-\frac{1}{4} \cot ^{2}|\theta|, \quad \tan ^{-1}\left(\frac{1}{2}\right) \leq|\theta|<\frac{\pi}{4} \\
& \frac{1-\frac{5}{8} \cot |\theta|}{1-\frac{1}{2} \cot |\theta|}, & \frac{\pi}{4} \leq|\theta|<\frac{\pi}{2} \\
& 1, & \frac{\pi}{2} \leq|\theta| \leq \pi
\end{array}
$$

As expected, opening with a square structuring element perfectly preserves all obtuse and right angles when $\alpha=0$.

The doubly compound operation close-open (CO) has exactly the same fractional corner preservation as opening, since the result of the first operation (closing) is the same as the original corner image for any $\theta$ and $\alpha$. 
Similarly, open-closing (OC) has the same $r(\theta, \alpha)$ as opening and CO because the second operation (closing) does not alter the result of the first operation (opening) in the case of a simple binary corner. Therefore, the fractional corner preservation in equation (5) is valid for opening, $\mathrm{OC}$, and $\mathrm{CO}$.

Although opening, $\mathrm{OC}$, and $\mathrm{CO}$ all have the same corner preservation characteristics according to this method, there may be other criteria that are important when deciding which filter to use in a particular situation. For instance, OC and CO typically provide more noise smoothing than opening or closing alone, and introduce less bias to the image. However, OC and $\mathrm{CO}$ are still biased with respect to the median [6]. To overcome this bias problem, averaging the corresponding morphological operators has been suggested [7-9]. The average of $\mathrm{OC}$ and $\mathrm{CO}$ is called the LOCO filter, and the average of opening and closing is known as the pseudomedian filter [10]. Since the outputs of OC and CO are identical for the original corner images, the LOCO filter has the same response as them, as given in equation (5). However, the pseudomedian filter is the average of two different outputs, and since these outputs are binary, the fractional corner preservation of the pseudomedian filter is given by:

$$
\mathrm{r}_{\text {pmed }}(\theta, \alpha)=\frac{1}{2}\left(1+\mathrm{r}_{\text {open }}(\theta, \alpha)\right)
$$

\subsection{Response of the Median Filter}

The fractional preservation for the median filter was determined numerically (in discrete space), using a 63 x 63 square filter window. The response of the median filter can be determined in continuous space for certain $\theta$ and $\alpha$, and these numbers may be used to check the simulation to see how close to the continuous case it is. Some of the known values of the median filter fractional preservation with $\alpha=0$ are given below. These values match those of the $63 \times 63$ simulation very well.

$$
\begin{array}{rlrl}
\mathrm{r}_{\text {med }}(\theta, 0)= & 0, & 0 \leq|\theta|<\tan ^{-1}\left(\frac{1}{2}\right) \\
\frac{2}{3}, & |\theta|=\frac{\pi}{4} \\
\frac{5}{4}-\frac{1}{2} \ln 4+\frac{1}{2} \ln 2, & |\theta|=\frac{\pi}{2} \\
1, & |\theta|=\pi
\end{array}
$$

\subsection{Comparison of Morphological and Median Filter Responses}

Fig. 3 is a plot of the fractional corner preservation of the opening, OC, CO, and LOCO operations compared with that of the pseudomedian and median filters for square structuring elements (filter windows). The functions are plotted in polar coordinates to give a visual indication of the angles of corners that are preserved and those that are destroyed. All curves in the figure are for $\alpha=0$ and $-\pi<\theta=\pi$. Note that for every angle $\theta>\tan ^{-1}(0.5)$, the LOCO filter preserves more of the corner than the median filter. The median filter does not preserve any corner exactly except the null case of $\theta=\pi\left(180^{\circ}\right)$, where the corner becomes a straight edge. The square LOCO filter, on the other hand, perfectly preserves corners with $\theta=\pi / 2\left(90^{\circ}\right)$ when $\alpha=0$.

Fig. 5 is another illustration of the same information. In this case, the attenuation of the corner is plotted in rectangular coordinates, with the angle of the corner (in degrees) as the xaxis and the attenuation $s(\theta, 0)$ in decibels as the y-axis. This way of plotting the information puts it in a form similar to those used for electrical filters, and by analogy one may define a "passband" and a "stopband" for the filters. For example, if we define the corner "passband" of a filter as the region where the attenuation is between 0 and $-3 \mathrm{~dB}$, the "passband" of the LOCO filter is approximately $\theta=43^{\circ}$. The median filter's "passband," in contrast, begins at about $\theta=48^{\circ}$. If we define the corner "stopband" to be where the attenuation is greater than $-10.67 \mathrm{~dB}$, then the "stopband" of the LOCO filter runs from 0 to about $30^{\circ}$, whereas for the median filter it runs from 0 to roughly $31^{\circ}$. The LOCO filter therefore has a wider "passband" and a sharper cutoff than the median filter, while the two filters 
have approximately the same "stopband." The pseudomedian filter has a "passband" of approximately $\theta=34^{\circ}$, but does not have a "stopband" according to the definition given above, since its maximum attenuation is -6 dB. We can see from this analysis that these filters are analogous to low pass filters, because they "pass" corners with angles above a certain value (the "cutoff" angle) and "stop" corners sharper than a given angle (which one may consider as having higher frequencies than more obtuse angles).

\subsection{Effect of Filter Window Shape}

Different window shapes may be used to improve the response of filters to corners. For instance, a plusshaped window is often used with the median filter when one wishes to preserve $90^{\circ}$ corners aligned with the lattice $(\alpha=0)$. Fig. 4 illustrates the corner response of the plus-shaped median filter compared to the squareshaped median filter. A closed form solution for this corner response, $r_{\mathrm{psm}}(\theta, 0)$, may be derived by geometry in continuous space, and is shown in the equation below. (Note that in continuous space a plus-shaped window has infinitely thin "arms," but the amount of the window that must be covered by 1's to result in an output of 1 is still easily determined.)

$$
\begin{array}{rlrl}
\mathrm{r}_{\mathrm{psm}}(\theta, 0)= & \tan |\theta|+\frac{1}{4} \frac{1}{1+\tan |\theta|}, & 0 \leq|\theta|<\tan ^{-1}\left(\frac{1}{2}\right) \\
& 1-\frac{1}{4} \frac{1}{\tan |\theta|(1+\tan |\theta|)}, & \tan ^{-1}\left(\frac{1}{2}\right) \leq|\theta|<\frac{\pi}{4} \\
& \left(\frac{7}{8}-\frac{1}{2} \cot |\theta|+\frac{1}{8} \frac{\tan |\theta|}{1+\tan |\theta|}\right)\left(\frac{2 \tan |\theta|}{2 \tan |\theta|-1}\right) & \frac{\pi}{4} \leq|\theta|<\frac{\pi}{2} \\
1, & |\theta| \geq \frac{\pi}{2}
\end{array}
$$

Fig. 6 shows the attenuation of the plus-shaped median filter in decibels. Its "passband" (as defined above) is corners with angles greater than about $29^{\circ}$, while its "stopband" is only 0 to $4^{\circ}$. The plus-shaped median filter therefore preserves corners significantly better than the square-shaped median filter, but its cutoff is not nearly as sharp, and it does not completely remove even very acute corners. This may sometimes be an advantage, since the plus-shaped median filter does preserve straight, thin lines aligned with either of its axes.

Morphological filters are very sensitive to structuring element shape. Square structuring elements are used widely and are very good at preserving obtuse corners, but the sharp $90^{\circ}$ corners of the structuring element can sometimes create a "stairstep" effect at edges in noisy images. Therefore, circular structuring elements are sometimes used. A closed-form solution for the corner response of opening, OC, CO, and the LOCO filter for a circle structuring element in continuous space can be found using geometry. However, the form of the solution is difficult, especially in the few degrees just above the angle where it exhibits complete cutoff. The tractable portions of the response $\mathrm{r}_{\text {open }}(\theta, 0)$ are: 


$$
\begin{array}{lll}
\mathrm{r}_{\text {open }}(\theta, 0)= & 0, & 0 \leq|\theta|<\beta \\
& 1-2(\cot |\theta|) \mathrm{f}(\theta), & \gamma \leq|\theta|<\frac{\pi}{4} \\
& 1-\left(\frac{1}{1-\frac{1}{2} \cot |\theta|}\right) \mathrm{f}(\theta), & \frac{\pi}{4} \leq|\theta|<\frac{3 \pi}{4} \\
& 1-\left(\frac{1}{2+\frac{1}{2} \tan |\theta|}\right) \mathrm{f}(\theta), & \frac{3 \pi}{4} \leq|\theta| \leq \pi \\
\text { where } & 5 \sin \beta=1+\cos \beta, \text { or } \beta \cong 22.6^{\circ} & \\
& 4 \sin \gamma=1+\cos \gamma, \text { or } \gamma \cong 28.1^{\circ} \\
& \mathrm{f}(\theta)=\frac{1}{16} \frac{\sin |\theta|}{1-\cos |\theta|}-\frac{\pi}{32}\left(1-\frac{|\theta|}{\pi}\right)
\end{array}
$$

The fractional preservation of the LOCO filter (and opening, OC, and $\mathrm{CO}$ ) with a round structuring element is compared to that with a square structuring element in Fig. 7. Values for the $6^{\circ}$ of angle missing in equation (9) were approximated by interpolation and verified by a discrete simulation. The circular structuring element preserves more acute corners than the square element, but does not perfectly preserve obtuse and right angles. Its "passband" is roughly $\theta>37^{\circ}$, and "stopband" approximately $\theta<27^{\circ}$. Therefore, the transition band of only about $10^{\circ}$ for the LOCO filter with a round structuring element is smaller than the transition bands for the LOCO and median filters with square windows.

\subsection{Effect of Corner Orientation Angle}

Another factor that can affect the corner response of a filter is the orientation angle of the corner with respect to the filter window, $\alpha$. For some filters, such as the plus-shaped median filter, this is a very important factor, whereas for others, such as the circular LOCO filter, it is virtually insignificant. Typically, a corner orientation angle of $\alpha=45^{\circ}$ produces the greatest difference in the filter response from the $\alpha=0$ response. The difference between the response of the square median filter at $\alpha=0$ and $\alpha=45^{\circ}$ is shown in Fig. 8. Most of the differences are very minor, and the "passband" and "stopband" are virtually unchanged. The $\alpha=45^{\circ}$ response was simulated the same way as the $\alpha=0$ response, with a $63 \times 63$ square window on a discrete lattice.

The LOCO filter (and opening, OC, and CO) shows a little more dependence on $\alpha$ than the median filter, as expected because of the importance of structuring element shape in morphological filtering. Fig. 9 illustrates the change in the response of the LOCO filter with a square structuring element when $\alpha$ changes from 0 to $45^{\circ}$. The transition and stop bands are very similar, but the "passband" of the LOCO filter shows significantly less preservation in the region $45^{\circ}<\theta<135^{\circ}$ for $\alpha=45^{\circ}$. The "passband" of the LOCO filter at $\alpha=45^{\circ}$ is approximately $\theta=44^{\circ}$, while the "stopband" is about $\theta=32^{\circ}$; both bands are within about $1^{\circ}$ of the LOCO filter bands with $\alpha=0$.

The plus-shaped median filter shows even more extreme changes in its response when the orientation angle changes from 0 to $45^{\circ}$. (At $\alpha=45^{\circ}$, the plus-shaped median is basically equivalent to a "cross-shaped" or "Xshaped" median at $\alpha=0$.) At $\alpha=45^{\circ}$, the plus-shaped median preserves less of the corner at nearly every angle, as shown in Fig. 10. The fractional preservation for $\alpha=45^{\circ}$ was determined numerically using a $25 \times 25$ window on a discrete lattice. The perfect preservation band for this filter changes from $\theta=90^{\circ}$ at $\alpha=0$ to $\theta=135^{\circ}$ at $\alpha=45^{\circ}$, just as for the LOCO filter. The "passband" for the plus-shaped median at $\alpha=45^{\circ}$ is about $\theta=31^{\circ}$, and the "stopband" is roughly $\theta=19^{\circ}$. The "passband" is similar, but the "stopband" is much wider than that of the plus-shaped median filter with $\alpha=0$. The transition band has shrunk to about $12^{\circ}$. 


\subsection{Summary of Examples}

Comparisons of four different filters at $\alpha=0$ and at $\alpha=45^{\circ}$ are shown in Figs. 11 and 12, respectively. The four filters are the square-shaped median, LOCO, and averaging filter and the plus-shaped median filter. Significant differences among the responses of these filters are easily observed. Using the "passband" and "stopband" definitions given earlier in the paper, we summarize the corner response information in Table I. The "perfect rejection" and "perfect preservation" bands correspond to regions of $\theta$ where $r(\theta, \alpha)=0$ and $r(\theta, \alpha)=1$ respectively.

Table I. Comparison of Filter Corner Responses.

\begin{tabular}{|c|c|c|c|c|c|c|}
\hline Filter & Rotation & $\begin{array}{c}\text { Perfect } \\
\text { Rejection } \\
(\mathrm{r}=0) \\
\theta=\end{array}$ & $\begin{array}{c}\text { "Stopband" } \\
\mathrm{s}<-10.7 \mathrm{~dB} \\
(\mathrm{r}<.293) \\
\theta=\end{array}$ & $\begin{array}{c}\text { Transition } \\
\text { Band } \\
\text { Width }\end{array}$ & $\begin{array}{c}\text { "Passband" } \\
\mathrm{s}>-3 \mathrm{~dB} \\
(\mathrm{r}>.707) \\
\theta=\end{array}$ & $\begin{array}{c}\text { Perfect } \\
\text { Preservation } \\
(\mathrm{r}=1)\end{array}$ \\
\hline Median & $\alpha=0$ & $27^{\circ}$ & $31^{\circ}$ & $17^{\circ}$ & $48^{\circ}$ & $180^{\circ}$ \\
(Square) & $\alpha=45^{\circ}$ & $19^{\circ}$ & $27^{\circ}$ & $22^{\circ}$ & $49^{\circ}$ & $180^{\circ}$ \\
\hline LOCO & $\alpha=0$ & $27^{\circ}$ & $31^{\circ}$ & $12^{\circ}$ & $43^{\circ}$ & $90^{\circ}$ \\
(Square) & $\alpha=45^{\circ}$ & $27^{\circ}$ & $32^{\circ}$ & $12^{\circ}$ & $44^{\circ}$ & $135^{\circ}$ \\
\hline Averaging & $\alpha=0$ & $0^{*}$ & $24^{\circ}$ & $47^{\circ}$ & $71^{\circ}$ & - \\
(Square) & $\alpha=45^{\circ}$ & 0 & $17^{\circ}$ & $69^{\circ}$ & $86^{\circ}$ & - \\
\hline Median & $\alpha=0$ & $-4^{*}$ & $4^{\circ}$ & $25^{\circ}$ & $29^{\circ}$ & $90^{\circ}$ \\
(Plus) & $\alpha=45^{\circ}$ & $14^{\circ}$ & $19^{\circ}$ & $12^{\circ}$ & $31^{\circ}$ & $135^{\circ}$ \\
\hline
\end{tabular}

${ }^{*} \mathrm{r}(\theta, 0) \rightarrow 0$ as $\theta \rightarrow 0$ for the averaging filter, but not for the plus-shaped median filter.

From Table I, we see that the square-shaped LOCO filter has the sharpest transition between corner removal and corner preservation at $\alpha=0$, and that its response changes the least when $\alpha$ changes to $45^{\circ}$. Thus it is the closest of these four filters to an "ideal" filter that has no transition band. However, there are usually many considerations other than just the response at corners that one must take into account when selecting a twodimensional filter. For example, the degree of noise reduction offered by the filters in Table I varies, and different filters excel in different types of noise. Also, the corner response characteristics of opening, OC, CO and the LOCO filter are all identical, despite obvious visual differences among the results of the filters in real image processing applications. Obviously, the corner response is not a complete description of the behavior of a filter.

Although the corner response technique is a valuable analysis tool, it does not indicate how one could design a filter with given corner response characteristics. The cutoff angles of filters vary with the angle of rotation $\alpha$ and the shape of the filter, but usually the cutoff angle cannot be drastically changed for a given filter. Also, the fractional preservation $\mathrm{r}(\theta, \alpha)$ only indicates the amount of area preserved, not any change in the shape of that area. Some filters, especially the median and morphological filters, achieve partial preservation of corners by changing their shape: the median filter rounds off sharp corners, while morphological operators tend to make corners look more like their structuring element. Thus a $45^{\circ}$ corner filtered by morphological open-closing with a square structuring element is $75 \%$ preserved $\left[\mathrm{r}\left(45^{\circ}, 0\right)=0.75\right]$, but the result may look like a more obtuse angle because this operation tends to clip corners to make them as close to right angles as possible.

Most of the above results were given for the "ideal" situation of continuous space or for very large discrete window sizes. Since most image filters use relatively small windows, it is important to see how the continuous corner response curves relate to those derived for more realistic filter sizes. Fig. 13 shows the corner response of a $5 \times 5$ square median filter compared to the $63 \times 63$ square result given earlier. Most of the differences between these two responses are discretization effects; that is, there are only a relatively small number of distinguishable angles in a $5 \times 5$ window. The overall trends and shape of the response for small windows are basically the same as for large windows or continuous space. 


\section{CONCLUSIONS}

We have introduced a new technique for analyzing the behavior of two-dimensional filters at corners. This technique quantifies a filter's preservation (or destruction) of corners of different angles and at different rotations. The response of a filter to corners at different rotations indicates how invariant the filter response may be to changes in the orientation of image features. Although there are many other factors usually considered when selecting a filter for a particular application, the corner response gives us valuable information that helps us understand the behavior of both linear and nonlinear filters. We have also shown that the square-shaped median and morphological filters (opening, $\mathrm{OC}, \mathrm{CO}$, and $\mathrm{LOCO}$ ) have a sharp transition between corner preservation and corner removal, and their response does not vary much with rotation. The responses of the plus-shaped median and averaging filter vary much more with the rotation of the corner, and usually have a wider transition between corner preservation and removal.

\section{REFERENCES}

1. N.C. Gallagher Jr. and G.L. Wise, "A theoretical analysis of the properties of the median filter," IEEE Trans. Acoust., Speech, Signal Process. 29(6), 1136-1141 (1981).

2. A.C. Bovik, T.S. Huang, and D.C. Munson, "The effect of median filtering on edge estimation and detection," IEEE Trans. Patt. Anal. Mach. Intell. 9, 181-194 (1987).

3. H. Longbotham and D. Eberly, "The WMMR filters: A class of robust edge enhancers," IEEE Trans. Signal Process. 41(4), 1680-1685 (1993).

4. J.P. Fitch, E.J. Coyle, and N.C. Gallagher, “The analog median filter,” IEEE Trans. Circuits Syst. 33(1), 94-102 (1986).

5. P. Maragos and R.W. Schafer, "Morphological filters-Part II: Their relations to median, order-statistic, and stack filters," IEEE Trans. Acoust., Speech, Signal Process. 35(8), 1170-1184 (1987).

6. R.L. Stevenson and G.R. Arce, "Morphological filters: Statistics and further syntactic properties," IEEE Trans. Circuits Syst. 34(11), 1292-1305 (1987).

7. J. Song and E.J. Delp, "A study of the generalized morphological filter," Circuits, Syst., Signal Process. 11(1), 227-252 (1992).

8. C.-H.H. Chu and E.J. Delp, "Impulsive noise suppression and background normalization of electrocardiogram signals using morphological operators," IEEE Trans. Biomed. Eng. 36(2), 262-273 (1989).

9. M.A. Schulze and J.A. Pearce. "Linear combinations of morphological operators: The midrange, pseudomedian, and LOCO filters." Proceedings of the 1993 IEEE International Conference on Acoustics, Speech, and Signal Processing, vol. V, pp. 57-60. Minneapolis: IEEE (1993).

10. W.K. Pratt, Digital Image Processing, 2nd ed. Wiley, New York (1991). 


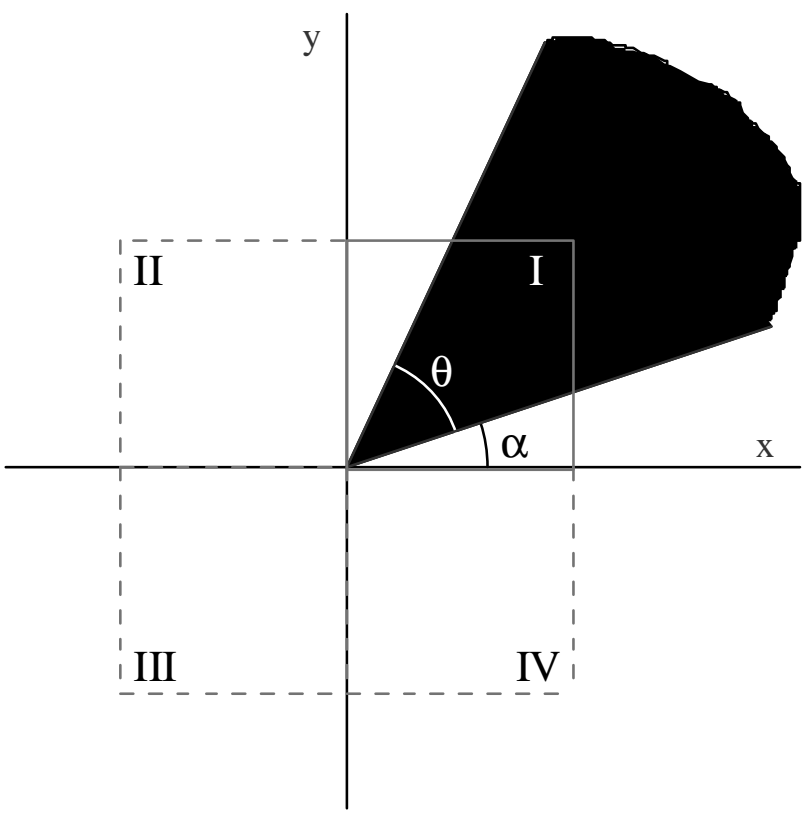

Fig. 1. Original image of corner with angle $\theta$ at rotation $\alpha$.

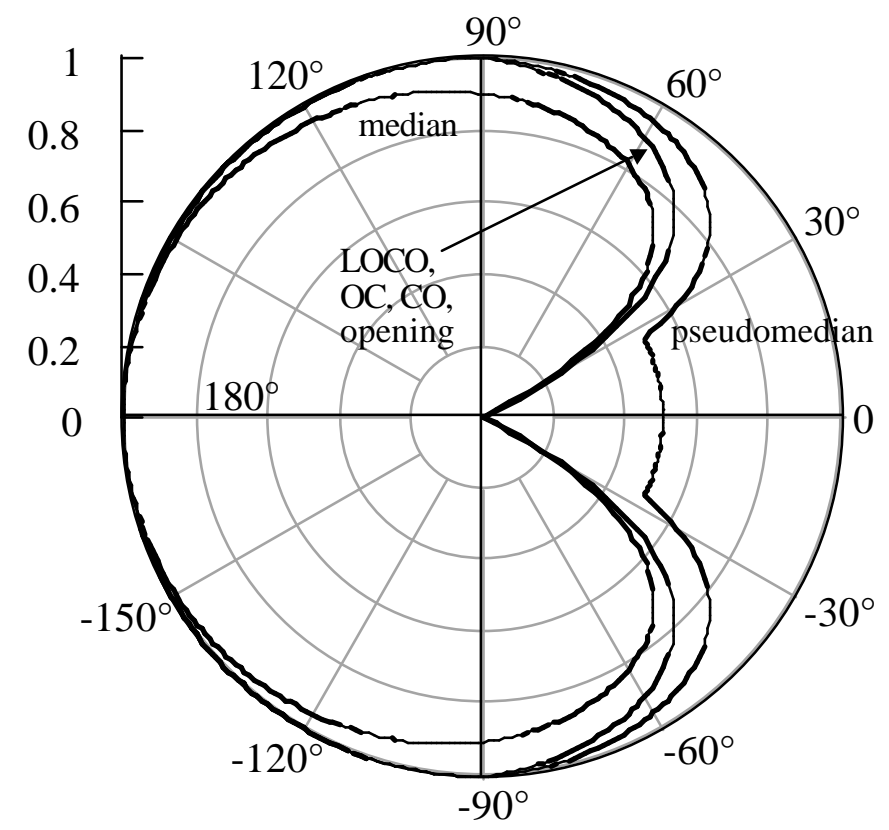

Fig. 3. Fractional corner preservation $r(\theta, 0)$ of morphological opening, $\mathrm{OC}, \mathrm{CO}$, and the LOCO filter compared to median and pseudomedian filters (square filter windows).

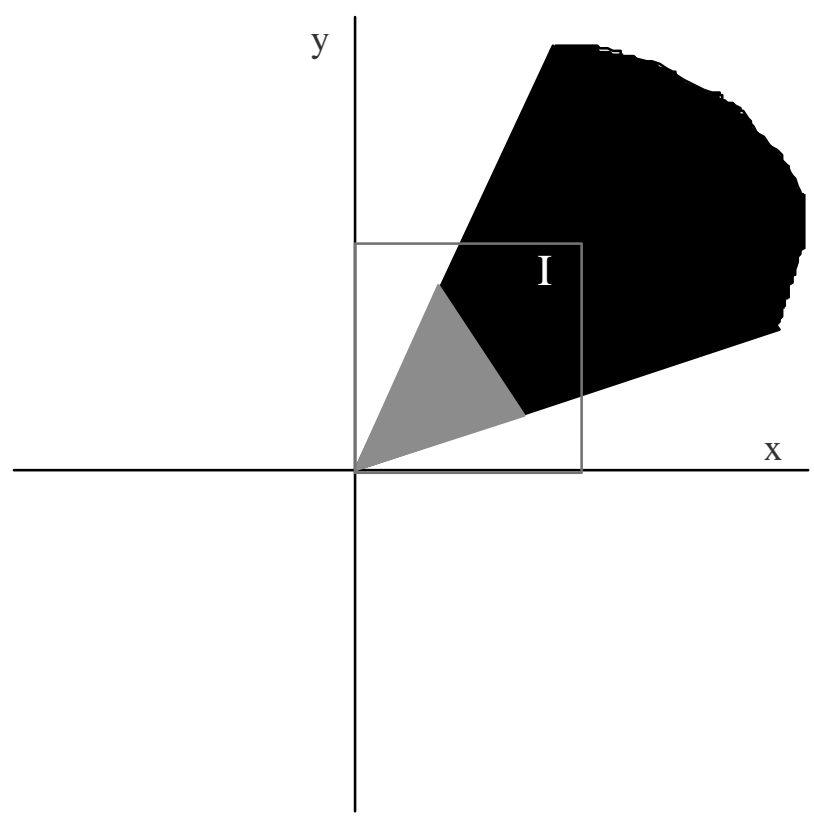

Fig. 2. Example filtered image of corner.

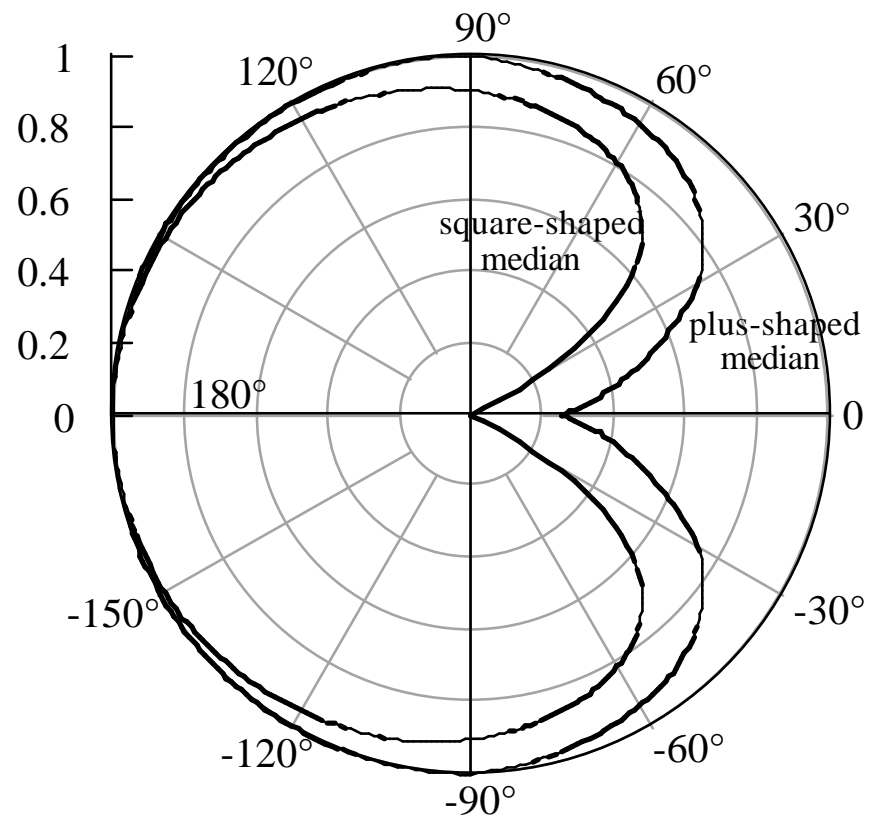

Fig. 4. Fractional corner preservation $r(\theta, 0)$ of the plus-shaped median filter compared to the square-shaped median filter. 


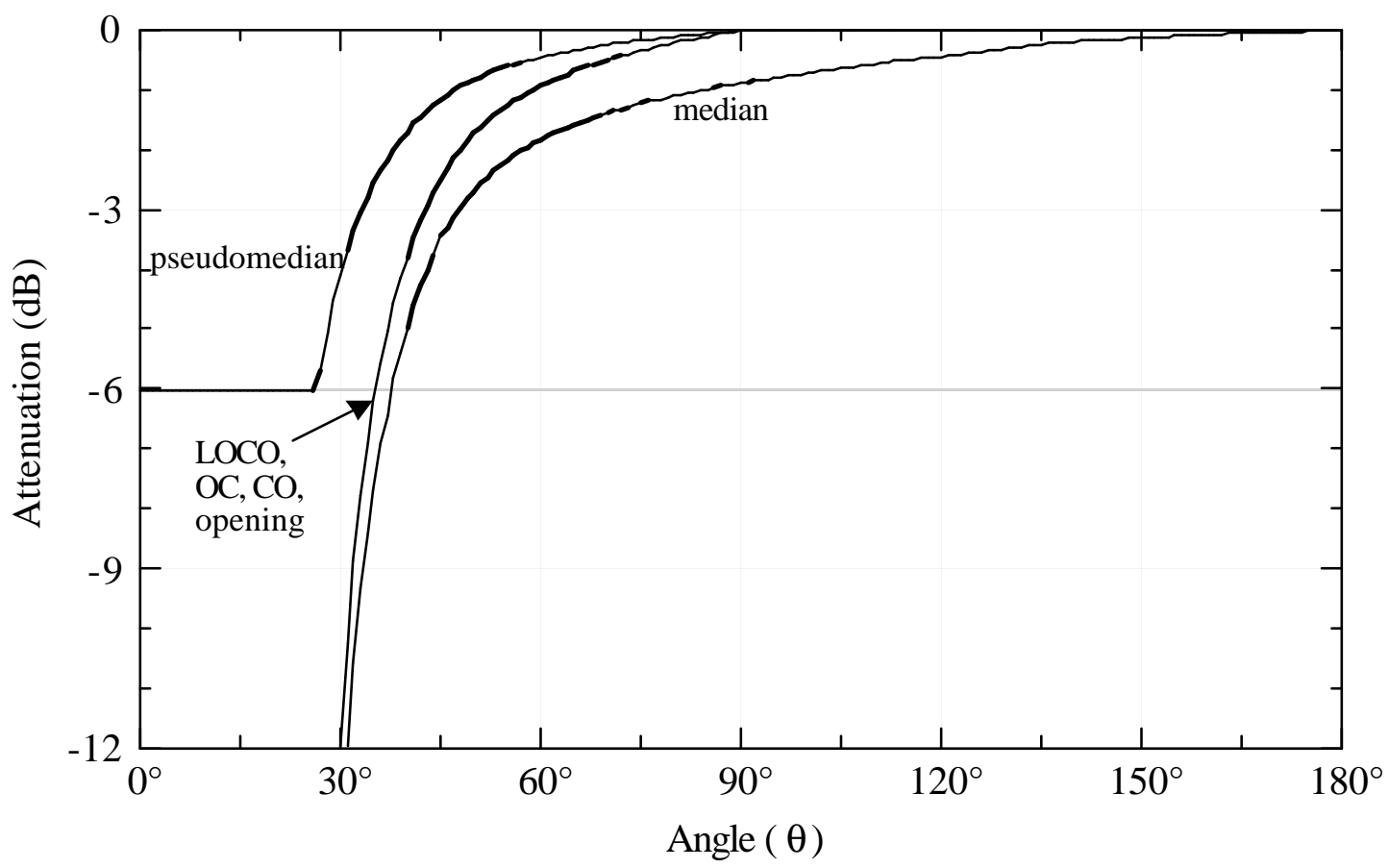

Fig. 5. Corner attenuation $\mathrm{s}(\theta, 0)$ of morphological opening, OC, $\mathrm{CO}$, and the LOCO filter compared to median and pseudomedian filters (square filter windows).

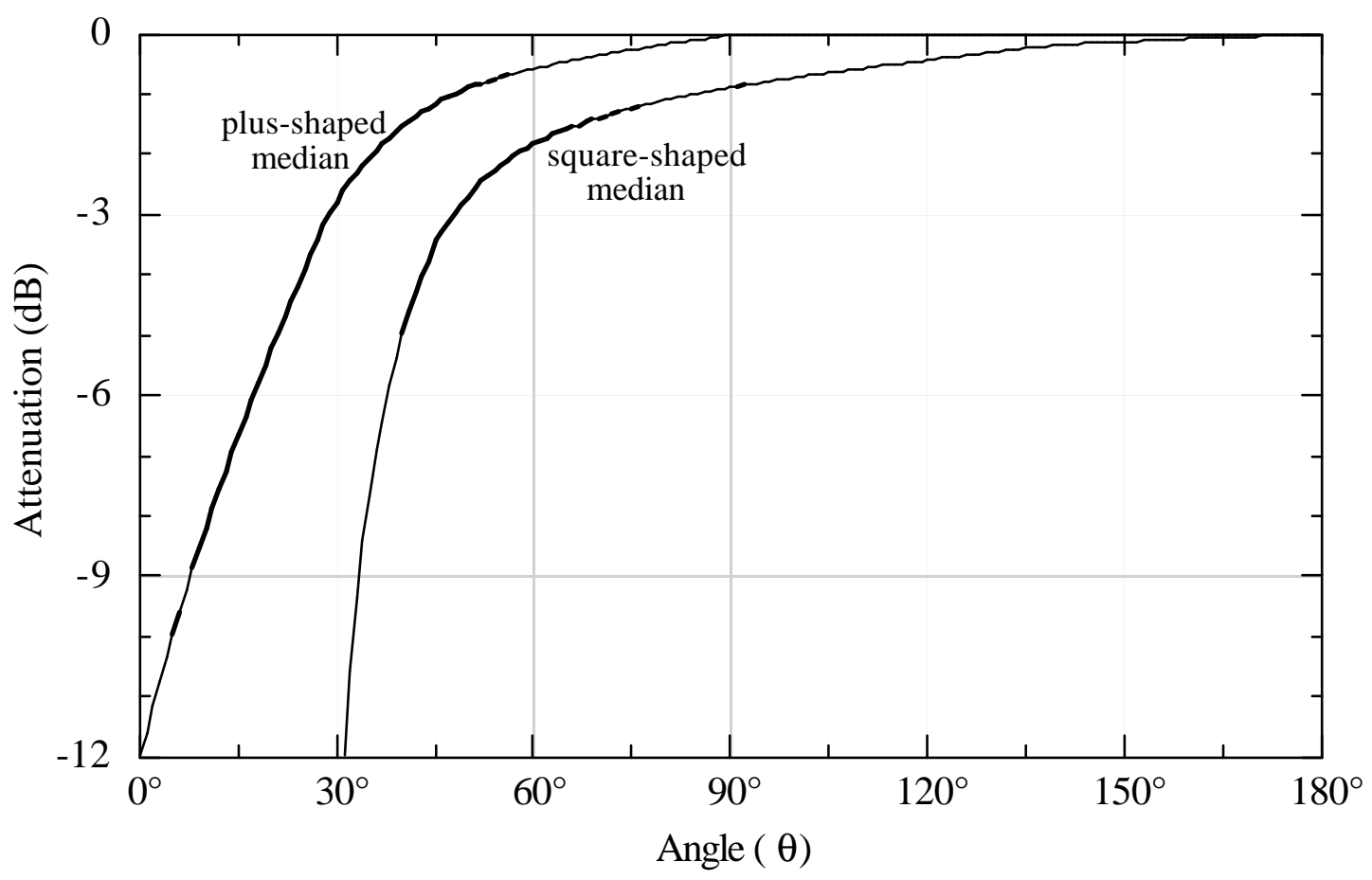

Fig. 6. Corner attenuation $\mathrm{s}(\theta, 0)$ of the plus-shaped median filter compared to the square-shaped median filter. 




Fig. 7. Fractional corner preservation $r(\theta, 0)$ of the LOCO filter with a circular structuring element compared to the LOCO filter with a square structuring element.

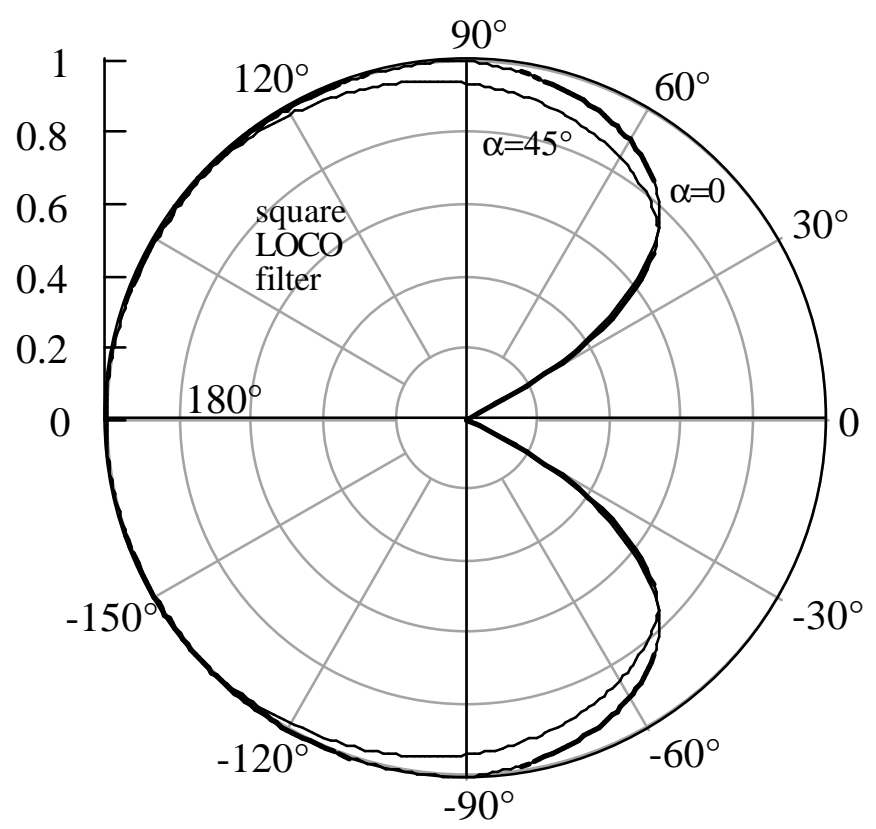

Fig. 9. Comparison of fractional corner preservation for the square LOCO filter at rotations $\alpha=0$ and $\alpha=45^{\circ}$.

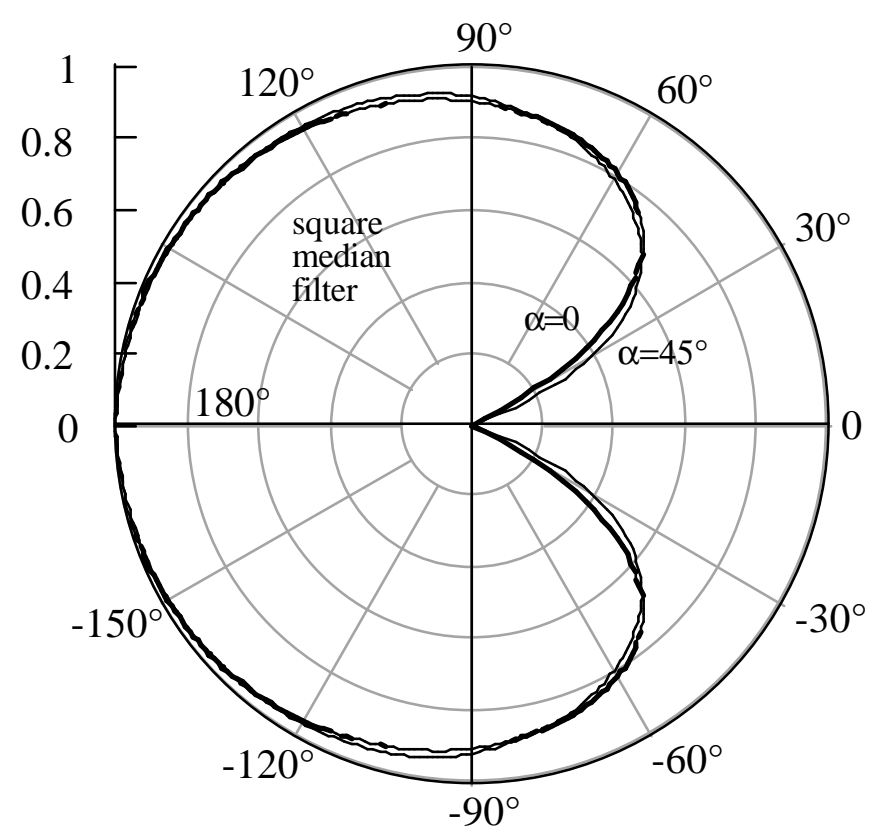

Fig. 8. Comparison of fractional corner preservation for the square median filter at rotations $\alpha=0$ and $\alpha=45^{\circ}$.

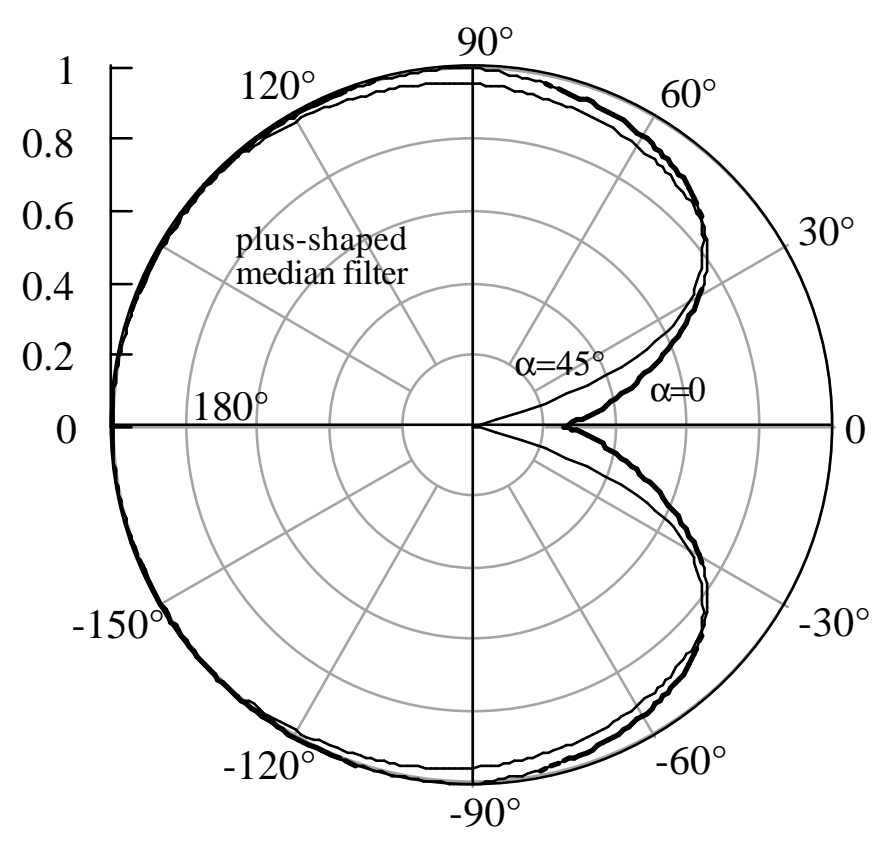

Fig. 10. Comparison of fractional corner reservation for the plus-shaped median filter at rotations $\alpha=0$ and $\alpha=45^{\circ}$. 




Fig. 11. Comparison of fractional corner preservation of square-shaped median, LOCO, and averaging filters and the plus-shaped median filter at $\alpha=0$.

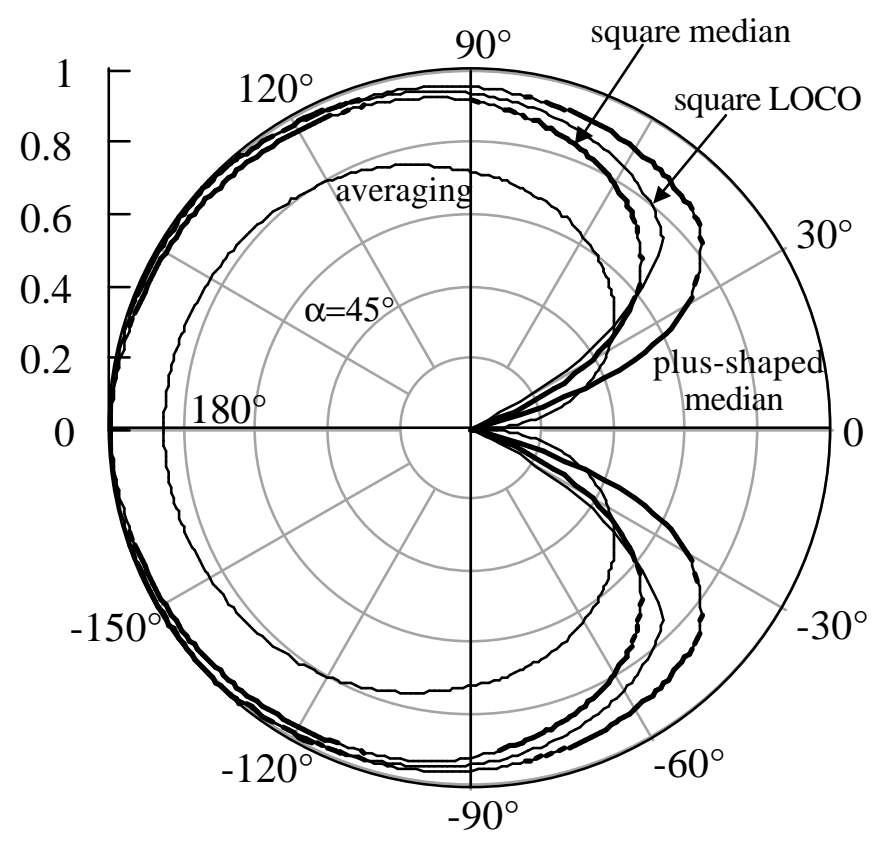

Fig. 12. Comparison of fractional corner preservation of square-shaped median, LOCO, and averaging filters and the plus-shaped median filter at $\alpha=45^{\circ}$.



Fig. 13. Comparison of fractional preservation of square median filter determined with $63 \times 63$ and $5 \times 5$ square windows. 